\title{
A Computational Multiagent Model of Bioluminescent Bacteria for the Emergence of Self-Sustainable and Self-Maintaining Artificial Wireless Networks
}

\author{
Nedjma Djezzar ${ }^{1,2}$, Iñaki Fernández Pérez ${ }^{2}$, NourEddine Djedi ${ }^{1}$ and Yves Duthen ${ }^{2}$ \\ ${ }^{1}$ University of Biskra, LESIA Laboratory \\ ${ }^{2}$ University of Toulouse, IRIT - CNRS - UMR5505 \\ E-mail: n.djezzar@univ-batna2.dz,inaki.fernandez-perez@irit.fr, noureddine.djed@gmail.com, yves.duthen@irit.fr
}

Keywords: artificial life, autonomy, distributed behavior, self-organization, self-regulation, regeneration abilities, quorum sensing, embedded metabolism, wireless networks, overhead messages

Received: July 7, 2018

\begin{abstract}
Biology is a rich source of inspiration in designing digital artifacts capable of autonomous, cooperative and distributed behaviors. Particularly, conceptual links can be established between (1) communication networks and (2) colonies of bacteria that communicate using chemical molecules. The goal of this paper is to propose a computational multiagent model of an interspecies bacterial communication system, termed quorum sensing, and analyze its self-sustainability and its self-maintaining ability to cooperatively form artificial wireless networks. Specifically, we propose a bottom-up agent-based approach combined with Ordinary Differential Equations, which abstract the intracellular dynamics, such as a proposed metabolism model that serves as a basis underlying self-sustainable networks. Results show that artificial bacterial cells have regeneration abilities in the light of random cell death and selected area for cell death, and a metabolism allowing them to exploit their own produced energy to cooperate at the population level to exhibit near-optimal self-organizing light-producing behaviors. The resulting artificial networks display several beneficial properties and could be used for the emergence of resistant wireless network topologies without the use of overhead messages.
\end{abstract}

Povzetek: Analizirano je komuniciranje med bakterijami, na osnovi katerih so razvite agentne metode za bolj odporna brezžična omrežja.

\section{Introduction}

Biology is a rich source of inspiration in designing artifacts capable of adaptive, distributed and autonomous behavior, which is one of the main goals of artificial life. In our works, we are interested in simulating the biological principles of self-regulation, to design artificial systems that display self-organized behaviors. One of the selforganizing principles of living organisms is that their units have the ability to communicate to help fulfill their goals. For instance, there is a growing belief that the robustness of biological systems is often derived from collective behaviors at a population level [1]. In the context of unicellular organisms, bacteria were considered for a long time to be independent unicellular organisms until 1979. Bacterial colonies of Vibrio-fischeri and Vibrio-harveyi were shown to be able to exhibit a cooperative lightemitting behavior, when the population reaches high cell densities [2]. This phenomenon is referred to as Quorum Sensing (QS). In fact, bacterial cells can communicate with each other by synthesizing, emitting, and sensing a signaling molecule known as an "autoinducer". When the autoinducer binds to the corresponding genetic receptor in a receiving cell, it triggers a regulatory response, which in turn results in the generation of more signaling molecules in the colony.

Communication is essential to any kind of coordinated parallel processes. The methods of communication have been investigated by the artificial life community in two contexts: unicellular and multicellular organisms. Multicellular approaches include artificial embryogeny [3] and morphogenetic engineering [4] models, which are developmental models that build an entire organism (a pattern or a morphology) from a single cell. The underlying idea is to model the behavior of how a single cell could reproduce and generate a predefined shape under specific constraints. Many multicellular models have been developed and used to solve different problems such as morphogenesis of French flags [5], [6].

Cell signaling in biological multicellular organisms results from a highly complex network of signaling pathways, including juxtracrine, paracrine and endocrine pathways, which are often abstracted into high-level mechanisms in computational models, and are difficult to thoroughly analyze. On the other hand, unicellular cell signaling mechanisms, such as quorum sensing, are relatively simple. Each bacterium produces and releases a signaling molecule that regulates gene expression over the bacterial colony when the population reaches high cell densities. Moreover, despite their sizes, bacteria have computational and evolutionary autonomous capabilities for self-replication and self-organization [7]. Indeed, compared to a cell of a multicellular organism, a bacterial cell is a mobile and autonomous entity that can grow and act independently at an individual level, and coordinate its behavior with other cells at a population level. 
In this paper, we propose the exploration of the unicellular approach, which provides several intrinsic beneficial properties, e.g. all the organisms are autonomous and share a single distributed communication system (QS).

To this end, we use a bottom-up agent-based approach and propose a cell-based model combined with Ordinary Differential Equations (ODEs), which includes a model of growth, a model of bioluminescence and a model of metabolism. We test our model in a set of experiments where we evaluate the sustainability and communication capabilities of bacterial colonies, their self-organized bioluminescence behavior, and their regeneration abilities. Our experiments reveal several insights into the cell behaviors to develop scalable artificial communication networks. In summation, our main contributions are:

i. As communication is essential to any kind of coordinated parallel processes in natural and artificial systems, we propose a computer-based simulation of a bacterial communication system (QS).

ii. Metabolism, as the biological process that allows for energy production, is crucial to any functional behavior. Thus, we propose a model of metabolism that allows bacteria to selfsustain, enabling them to grow, divide, and communicate using their own self-produced energy.

iii. Bacterial cells in our model are able to cooperate at the population level to exhibit near-optimal light-producing behaviors using our proposed model of bioluminescence.

iv. Our unicellular communication model possesses emerging abilities of regeneration in two cases: random distributed cell death, and particular cell death in a specific region of the colony.

v. Bioluminescence is conceived as a basis underlying cooperative artificial network formation. The emergent communication network displays beneficial properties: selfreproduction of the network nodes (cell division), cooperative formation of the network links (QS), and autonomy via self-sustaining network nodes (metabolism). These intrinsic network properties lead to the evolution of cooperation toward common goals, such as increasing the number of networked cells.

vi. A parallel is made between wireless networks and simulations of bacteria colonies that communicate using QS molecules. The resulting artificial network could be potentially used for the emergence of autonomous networks that can address issues such as limiting the use of configuration messages commonly known as overhead messages, which are a key factor for the development of new self-organized networks [8].

Finally, we believe that the abstract representation of complex interactions among QS molecules would contribute to enrich our understanding of biological microbial communities, which may provide further insights for novel design techniques.

The rest of this paper is organized as follows. Section 2 presents the related works and the problem statement. Our cellular model for quorum sensing, growth, metabolism, and bioluminescence is described in Section 3. Our artificial communication network model is presented in Section 4. The simulation results are evaluated and discussed in Section 5 and 6. Section 7 concludes the paper.

\section{Related works and problem statement}

The related works are grouped in three subsections: quorum sensing, regeneration and bioinspired wireless networking for self-organizing network topologies.

\subsection{Quorum sensing}

Quorum sensing, as a simple and powerful biological communication system, has attracted the interest of interdisciplinary research groups. In the field of bioinspired systems, QS has been investigated from different perspectives, including artificial ecosystems [9], membrane computing [10], digital evolution [11], swarm robotics [12], logic computing [13], dynamic clustering [14], synthetic systems [15], bioinspired agent-based modeling [16] and control [17], and game theory [18].

On the one hand, in the field of artificial life, only a small number of works have investigated digital simulations of QS considering metabolism and bioluminescence. While in the seminal works of [19] and [20], a QS simulation was proposed, their model did not consider bacterial metabolism for energy production and consumption. Since energy is vital to any physiological process in living organisms, in this paper we include such a model of metabolism in our simulations. Furthermore, we propose a computational model of bioluminescence using the same QS model.

Additionally, few works have investigated the analogy between QS simulations and communication networks: in our work, we establish a conceptual link between QS and artificial communication networks. In [21], a QS-based communication network model was proposed, which used autoinducer molecules for communication. In our work, we address a similar problem using a network-centric approach, but we use a light-based communication protocol instead of autoinducers, because:

- propagation of light is less limited by distance than a signaling molecule,

- the different intensities of light are favorable for hierarchical structures, with several cell types: super-spreaders of light called wild-type 
cooperators, simple spreaders called cooperators, and non-bioluminescent cells which do not spread light, called cheaters. Hierarchical structures are known to be beneficial for the optimization of the network resources [22].

\subsection{Regeneration abilities}

Regeneration is the ability of an organism, unicellular or multicellular, to resist damage by re-growth and renewal of dead cells. Hardware regeneration, often referred to as self-repairing, is a technique that allows electronic systems to reconfigure themselves if a part of a unit breaks down. Bioinspired developmental models are usually used. This part of electronics is commonly called embryogenics. Miller developed a self-repairing system, [23] [24] using cellular automata and Cartesian Genetic Programming. His model can generate a French flag able to reconstruct itself when the environment randomly moves the cells. This work has been reproduced by Liu [25] on a reprogrammable electronic ship, showing faulttolerance abilities.

As additional models about regeneration of artificial multicellular organisms have been proposed, we can cite the regeneration of dead cells in previous studies: the twodimensional and three-dimensional shapes of Fleischer [26] , the star fish of Cussat-Blanc et al. [27], and the bat of Djezzar et al. [28]. In [29], an efficient cell-to-cell communication mechanism that allows the maintenance of Planarian worm-like shapes was introduced. The efficiency of the model has been verified in the light of random cell death. However, regeneration after a selected area for cell death was presented as a challenge that was not raised by the proposed mechanism.

On unicellular artificial life simulation models, regeneration is less studied. Fewer works exist on regeneration and resistance of unicellular organisms. In this paper, we present a unicellular model of communication with emerging abilities of regeneration studied in two cases: (1) in the light of random cell death, and also (2) a selected area for cell death due to factors such as the action of an antibiotic or damage on a specific area of the unicellular-based structure. These regeneration abilities are beneficial for the development of selforganizing network topologies with self-maintaining features.

\subsection{Bioinspired wireless networking for self-organizing topologies}

One of the major challenges is topology when wireless networks are designed. The network topology or physical placement of nodes is the base infrastructure that can intensely affect the entire network performance. Indeed, a careful node placement in a wireless networks can be an effective optimization means for coping with many resources limitation problems such as energy, location data requirements, computation time, and especially overhead communications, such as hello configuration messages. Overhead messages present common issues in designing a network topology, particularly:
- computation time and energy.

- security vulnerability. For example, during the exchange of overhead (hello) messages, a listener node can save important information on the network's structure such as the location of cluster heads.

- self-adaptation when the topology is subjected to failures. A flaw in the topology necessitates the relaunching of overhead controls as well as human intervention.

Nature and bio-inspired mechanisms of selforganization could present an efficient solution to reduce overhead controls in designing a wireless network topology. Indeed, artificial and swarm intelligence have a long history of use as bioinspired alternative approaches able to transform natural patterns of collective behavior into useful models for self-organizing network topologies. In fact, researchers have proposed models inspired from ants [30], honeybees [31], fireflies [32], [33], Boids of Reynolds [34], etc. A survey on bioinspired networking is presented in [8]. Most of the existing approaches, bioinspired or not, require geographic data on nodes, such as position, and overhead messages.

Considering the wireless network as a biological system is very interesting. It is connected to the goal of this paper. To achieve this goal, artificial life and particularly morphogenetic engineering is an original promising idea to emulate self-regulation, cooperation and regeneration capabilities of colonies of bacteria for the emergence of network topologies presenting these features.

Consequently, in this paper we propose an artificial life simulation model of bacteria QS for the emergence of wireless network topologies, and for the purpose of:

- eliminating overhead messages.

- regeneration, resilience, and tolerance abilities, especially when the network is subjected to failures or external attacks.

\section{Cellular dynamics model}

Our cells are bacterial agents that evolve in a twodimensional environment and have the ability to sense the environment (taking up substrates and autoinducers), grow, divide and survive. They possess a QS genetic controller circuit allowing them to coordinate their cellular communication with other cells. Moreover, they are able to synthesize light via a proposed model of bioluminescence and have a metabolism allowing them to accomplish all of these actions.

\subsection{Quorum sensing}

To simulate cell-to-cell communication in heterogeneous microbial communities, we use a generic $L u x I / L u x R$ QS language that is employed by over thirty species of Gramnegative bacteria [2]. All LuxI/R systems are mediated by autoinducers, such as acylated homoserine lactone $(A H L)$. Explicitly, the autoinducer molecule is synthesized by the 
synthase luxI homologs, $\operatorname{LuxR}$ is a receptor that can bind the $A H L$ molecules, and the $L u x R-A H L$ complex activates the transcription of the downstream operon.

The molecular regulation network of a bacterial agent is based on the empirical ODE-models proposed by [19] and [20]. This model uses two positive feedback loops (Figure 1). The autoinducer AHL $(A)$ and the receptor LuxR $(R)$ form a dimerized complex $(C)$ that regulates the expression of both luxI and luxR genes, which produces more $A H L$ molecules and $L u x R$ receptors, respectively. The following equations describe the molecular dynamics of this genetic circuit:

$$
\begin{gathered}
\begin{array}{c}
\frac{d[A]}{d t}=C_{A}+\frac{k_{A}[C]}{K_{A}+[C]}-k_{0}[A]-k_{1}[R][A]+k_{2}[R A] \\
\quad-p_{e}[A]+p_{a}\left[A_{e}\right], \quad(1)
\end{array} \\
\begin{array}{c}
\frac{d[R]}{d t}=C_{R}+\frac{k_{R}[C]}{K_{R}+[C]}-k_{3}[R]-k_{1}[R][A] \\
\quad+k_{2}[R A], \quad(2)
\end{array} \\
\begin{aligned}
\frac{d[R A]}{d t}= & k_{1}[R][A]-k_{2}[R A]-2 k_{4}[R A]^{2}+2 k_{5}[C],(3)
\end{aligned} \\
\begin{array}{c}
\frac{d[C]}{d t}=k_{4}[R A]^{2}-k_{5}[C],(4) \\
\frac{d\left[A_{e}\right]}{d t}=\sum_{\text {bact }}\left(p_{e}[A]-p_{a}\left[A_{e}\right]\right)+D \nabla^{2}\left[A_{e}\right],
\end{array}
\end{gathered}
$$

where the notation $[X]$ represents the concentration of a particular molecular species $X$ and $R A$ is the $\operatorname{LuxR}-$ $A H L$ complex. $A_{e}$ is the extracellular concentration of $A$ sensed from the environment. $p_{e}$ and $p_{a}$ are emission rate and absorption rate of $A$ and $A_{e}$, respectively. $C_{A}$ and $C_{R}$ represent the basal level transcription of $A$ and $R$, respectively.

\subsection{Growth}

For simplicity, cells grow through the substrate-dependent growth model of Monod [35]. In the model of Monod, the specific growth rate $(\mu)$ of a bacterium biomass $(X)$ depends on the substrate concentration $(S)$. The equation is given by:

$$
\mu=\mu_{\max } \cdot \frac{S}{S+K_{S}}
$$

where $\mu_{\max }$ is the maximum growth rate and $k_{s}$ is the substrate affinity (the value of $S$ when $\mu / \mu \max =0.5$ ). These two parameters are assumed to be constant but depend on strain and environmental conditions. Using the specific growth rate $(\mu), d[X] / d t$ is calculated as follows:

$$
\frac{d[X]}{d t}=X \cdot \mu
$$

To calculate the specific energy requirement rate $\left(q_{\text {ATP }}\right)$ for cell growth, Stouthamer and Bettenhausen [36] introduced Eq. 8 and used the energetic growth yield coefficients $\left(Y_{X / A T P}\right)$. This parameter is assumed to be constant and represents the cell mass synthesized $(X)$ per unit of energy generated $(A T P)$. The equation is given by:

$$
q_{A T P}=\frac{\mu}{Y_{X / A T P}}
$$

We note that the energy ( $A T P$ ) consumption due to the cell growth is subtracted from the total energy of the cell.

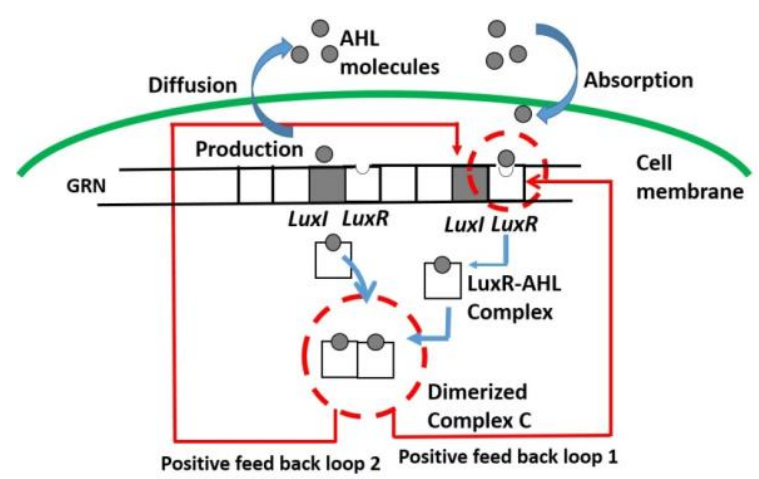

Figure 1: Dynamics of a LuxI / LuxR quorum sensing. The LuxI synthesize the autoinducer molecule $(A H L)$. The receptor $L u x R$ can bind the $A H L$ molecule to form a complex $\operatorname{LuxR}-A H L$. The dimerized complex composed of two $\operatorname{LuxR}-A H L$ complexes, regulates the expression of both $L u x R$ (positive feedback loop 1), and LuxI (positive feedback loop 2), which produces more LuxR receptors and $A H L$ molecules, respectively.

\subsection{Bioluminescence}

In general, bioluminescence is a light-producing reaction catalyzed by a luciferase. Luciferase is a photoprotein enzyme that transforms a light-producing substrate called luciferin into light. The process requires the presence of other substances, like oxygen and adenosine triphosphate $(A T P)$. For simplicity, oxygen is assumed to exist in abundance in the environment. Therefore, the enzymatic reaction can be written in the form of a bi-molecular reaction that involves an enzyme $(E)$, binding to a substrate $(S)$ to form a complex $(E S)$, which in turn releases a product $(P)$, regenerating the original enzyme. This may be represented as follows:

$$
E+S \stackrel{K_{f}}{\stackrel{K_{r}}{\rightarrow}} E S \stackrel{K_{\text {cat }}}{\longrightarrow} E+P,
$$

where $K_{f}$ is the forward rate, $K_{r}$ is the reverse rate, and $K_{c a t}$ is the catalytic rate. By applying conservation constraints of the material and assuming that the concentration of enzymes is very low in comparison with the metabolite concentration, the equation describing this reaction is as follows:

$$
\frac{d[P]}{d t}=\frac{K_{c a t}[E][S]}{\frac{K_{r}+K_{c a t}}{K_{f}}+[S]},
$$

By setting: $K_{L}=\frac{K_{r}+K_{c a t}}{K_{f}}$ and $P_{\max }=K_{c a t}[E]$, we obtain the following equation:

$$
\frac{d[P]}{d t}=\frac{P_{\max }[S]}{K_{L}+[S]}
$$

where $P_{\max }$ represents the maximum production rate and $K_{L}$ is the concentration of $S$ at which the reaction rate is at half-maximum.

In the case of bioluminescent bacteria, the bacterial luciferase is encoded and synthesized by the lux operon. The transcription of the lux operon is activated by the LuxR - AHL dimerized complex $(C)$ as shown in Figure 


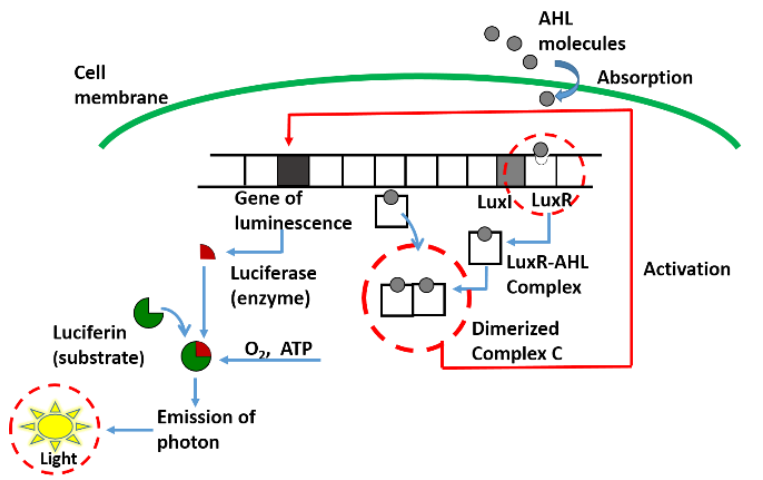

Figure 2: Light production regulated by a $\operatorname{luxI} / \operatorname{luxR} \mathrm{QS}$.

2. The bacterium produces light only at high cell density (i.e. only when a quorum is met). At low cell densities, even with higher concentration of the luciferin substrate, bacterial cells do not produce light. Then, we assume that: (1) the substrate exists abundantly in the cell cytoplasm and (2) the dimerized complex $C$ that controls the synthesis of the luciferase enzyme is assumed to be a determining factor. Therefore, we model the cell light production as a function of the dimerized complex $C$. Hence equation Eq. 11 is modified to:

$$
\frac{d[L]}{d t}=\frac{L_{\max }[C]}{K_{L}+[C]}
$$

where $L$ is the light production rate, $L_{\max }$ is the maximum light production rate, and $K_{L}$ is the concentration of $C$ at which $L$ is at half-maximum. Bioluminescence is expressed as the accumulation of the green fluorescent protein $g f p$. The $g f p$ is incremented at each time step according to the light production rate $L$. The more $g f p$ a cell accumulates, the greener the cell becomes.

\subsection{Metabolism}

Metabolism is the process that describes energy production and consumption [37]. In our model, metabolism is calculated by subtracting the energy spent to grow, divide, produce light, or survive (maintenance energy) from the total energy of the organism (sum of basal energy and energy produced from substrate). Figure 3 represents material and energy inputs and outputs of the cell metabolism. ATP is the molecule that stores and transports energy in living organisms. We model the bacterial $A T P$ cycle as follows:

$$
\begin{gathered}
\Delta A T P=\left(A T P_{0}+E . A T P_{S}-A T P_{G}-A T P_{D}-A T P_{L}\right. \\
\left.-A T P_{M}\right)(\Delta t),(13)
\end{gathered}
$$

where:

- $\triangle A T P$ is the total energy change of the organism.

- $A T P_{0}$ is the basal energy.

- $A T P_{S}$ is the substrate energy. It represents the energy produced from metabolized substrates. This term is calculated as follows:

$$
\frac{d\left[A T P_{S}\right]}{d t}=Y_{A T P / S} \cdot \frac{d[S]}{d t},
$$

where $Y_{A T P / S}$ is the energetic substrate yield. It represents the amount of $A T P$ produced per unit of substrate.

- $E$ is the substrate metabolism efficiency (equal to $40 \%$ ). This is because only 40 to $50 \%$ of the energy stored in a carbon substrate is converted to biological energy (ATP). The rest is released as heat (Figure 3).

- $\operatorname{ATP}_{G}$ is the growth energy. It represents the energy consumption due to the cell growth. The consumption of ATP due to the cell growth, at each time step, is given by:

$$
\frac{d\left[A T P_{G}\right]}{d t}=q_{A T P} \cdot X,
$$

Using Eq. 8, Eq. 15 becomes:

$$
\frac{d\left[A T P_{G}\right]}{d t}=\frac{\mu}{Y_{X / A T P}} \cdot X,
$$

Using Eq. 7, Eq. 16 becomes:

$$
\frac{d\left[A T P_{G}\right]}{d t}=\frac{1}{Y_{X / A T P}} \cdot \frac{d[X]}{d t} \text {, }
$$

- $\quad A T P_{D}$ is the division energy. It

represents the energetic cost of cell division and is equal to $0.5 \mu M$.

- $A T P_{L}$ is the light energy. It represents the energy used to produce light. We use a similar equation to Eq. 17 to calculate $A T P_{L}$. This is given by:

$$
\frac{d\left[A T P_{L}\right]}{d t}=\frac{1}{Y_{L / A T P}} \cdot \frac{d[L]}{d t},
$$

where $Y_{L / A T P}$ is the energetic light yield coefficient.

- $\quad \operatorname{ATP}_{M}$ is the maintenance energy (equal to $0.25 \mu \mathrm{M}$ ).

\subsection{Parameters}

Table 2 lists the parameters used in our model. QS parameter values are adopted from [20].

\begin{tabular}{|c|l|l|}
\hline Parameter & Value & Unit \\
\hline$p_{e}$ & 0.025 & - \\
\hline$p_{a}$ & 0.025 & - \\
\hline$\mu_{\max }$ & 0.034 & $\mathrm{fl} / \mathrm{min}$ \\
\hline$K_{S}$ & 1 & $\mu \mathrm{Mol}$ \\
\hline$Y_{X / A T P}$ & 0.034 & $\mathrm{fl} / \mu \mathrm{Mol}$ \\
\hline$L_{\max }$ & $2.0 \mathrm{e}-1$ & $R L U / \mathrm{min}$ \\
\hline$K_{L}$ & $2.1 \mathrm{e}-7$ & $\mu \mathrm{Mol}$ \\
\hline$A T P_{0}$ & 1000 & $\mu \mathrm{Mol}$ \\
\hline$Y_{A T P / S}$ & 1000 & - \\
\hline$Y_{L / A T P}$ & 0.135 & $R L U / \mu \mathrm{Mol}$ \\
\hline$T_{L}$ & 0.20 & - \\
\hline
\end{tabular}

Table 1: Model parameters. 


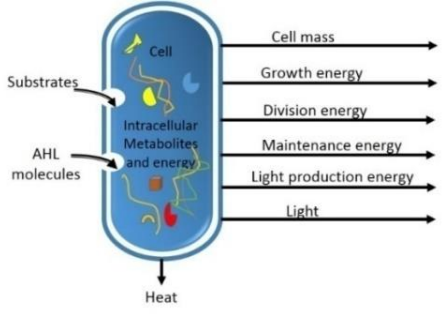

Figure 3: Material and energy inputs and outputs of the cell metabolism.

\section{Artificial wireless network}

QS is intrinsically a wireless communication system. Practically, traditional wireless networks can connect heterogeneous devices without physical links. Similarly, within microbiomes, different types of bacteria can establish a wireless molecular communication network without previously established paths.

However, the QS-based communication network is different from a classical wireless network in the sense that bacterial cells do not have IP addresses and the signals that they convey (autoinducers) do not have specific destinations encoded in the signals. This means that QS establishes communication without access to the location of cells. We want to take advantage of this property for the emergence of a network topology without using data location (cell position) and overhead messages. To do so, our network is based only on the internal dynamics of nodes, i.e. intracellular factors, and we use light as a modeling choice for the following reasons:

1. Light is a self-organizing cooperative behavior that emerges from QS dynamics.

2. The different light productivities, i.e. intensities, strong, (hyper-luminescent cells), medium, (luminescent cells), and null (non- luminescent cells), permit a network topology with a certain hierarchy, very suitable for networks, because it optimizes the network resources [22].

3. Light as an internal factor $(g f p)$ allows self-selection of the node types without a centralized control. Indeed, a simple local test of the value of the $g f p$ at a cell allows for the determination of the node's type.

4. Light is an internal factor, but it has a beneficial external half-side effect. It is visible by other nodes and therefore can be sensed, for example, by a photosensitive sensor node. A signaling molecule such as autoinducer does not have this characteristic. The internal concentration of a signaling molecule inside a node is not visible by other nodes.

5. The strength of the emitted signal in a traditional wireless network may be analogous to the light intensity.

6. The sensitivity of the receiver in a traditional wireless network is analogous to the concentration of the signal receptor $(\operatorname{Lux} R)$.

Based on the above observations, we propose a QS network based only on intracellular factors. Figure 4 shows the network node types and explains how the network links are established to from a network topology.
The following subsections explains the network links and nodes.



Figure 4: Establishment of the bacteria network topology. (a) WT-cooperator bacteria can connect to cooperators and cheaters. (b) Cooperator bacteria can connect to cheaters and can receive signals from WT-cooperators. (c) Cheaters cannot connect to WT-cooperators and cooperators but can receive signals. The direction of the link shows how the link is established. (d) The more gfp a cell accumulates, the greener the cell becomes, and the cell switches to the other cell type. Cheaters can switch to cooperators, and cooperators can switch to WTcooperators.

\subsection{Network links}

A directed link is established from bacterium A to bacterium $\mathrm{B}$ under two conditions:

- The light signal concentration ( $g f p /$ volume $)$ inside bacterium $\mathrm{A}$ is larger than that of bacterium $\mathrm{B}$.

- The bacterium B is a sensitive receiver. A bacterium is considered to be a sensitive receiver to light if its concentration of $\operatorname{LuxR}$ is above an activation threshold $T R$.

The first condition specifies the link direction. It represents the fact that there is a descending light gradient from bacterium A to Bacterium B. The second condition ensures that bacterium $\mathrm{B}$ is able to receive the signal.

\subsection{Network nodes}

QS is a cooperative effort of a bacterial population in which certain bacteria do not participate. The nonparticipating bacteria are usually called cheaters [38].

In our work, a node is an abstraction of a bacterium cell. Since, within microbiomes, different type of cooperators and cheaters tend to coexist in collaboration or in conflict with one another [39], [40], we adopt a similar biological terminology to define the node's types of our artificial wireless network.

To account for different light productivity, we classify the network nodes into three categories based on the intracellular intensity of light (gfp/volume). More precisely:

- nodes with $g f p / v o l u m e>20$ are up-regulated cells with high productivity of light (they are able to produce light at a high intensity). The nodes in this category are super spreaders that can send light to all 
the other types of nodes. They are considered to be wild-type cooperators (WT-cooperators).

- Nodes with $0<g f p / v o l u m e<20$ are considered to be cooperators. These nodes are down regulated cells that can receive light from all WT-cooperators but do (femtoliters). To support the survival and growth of cells, we assume a constant nutrition concentration $S=$ $10 \mu$ Mole. In Figure 5a-c, cells that are close to the nutrient source are able to sense and absorb substrates. As a cell takes in substrates, it grows until it doubles its volume to $V=3.14 f L$, at which point it divides. When

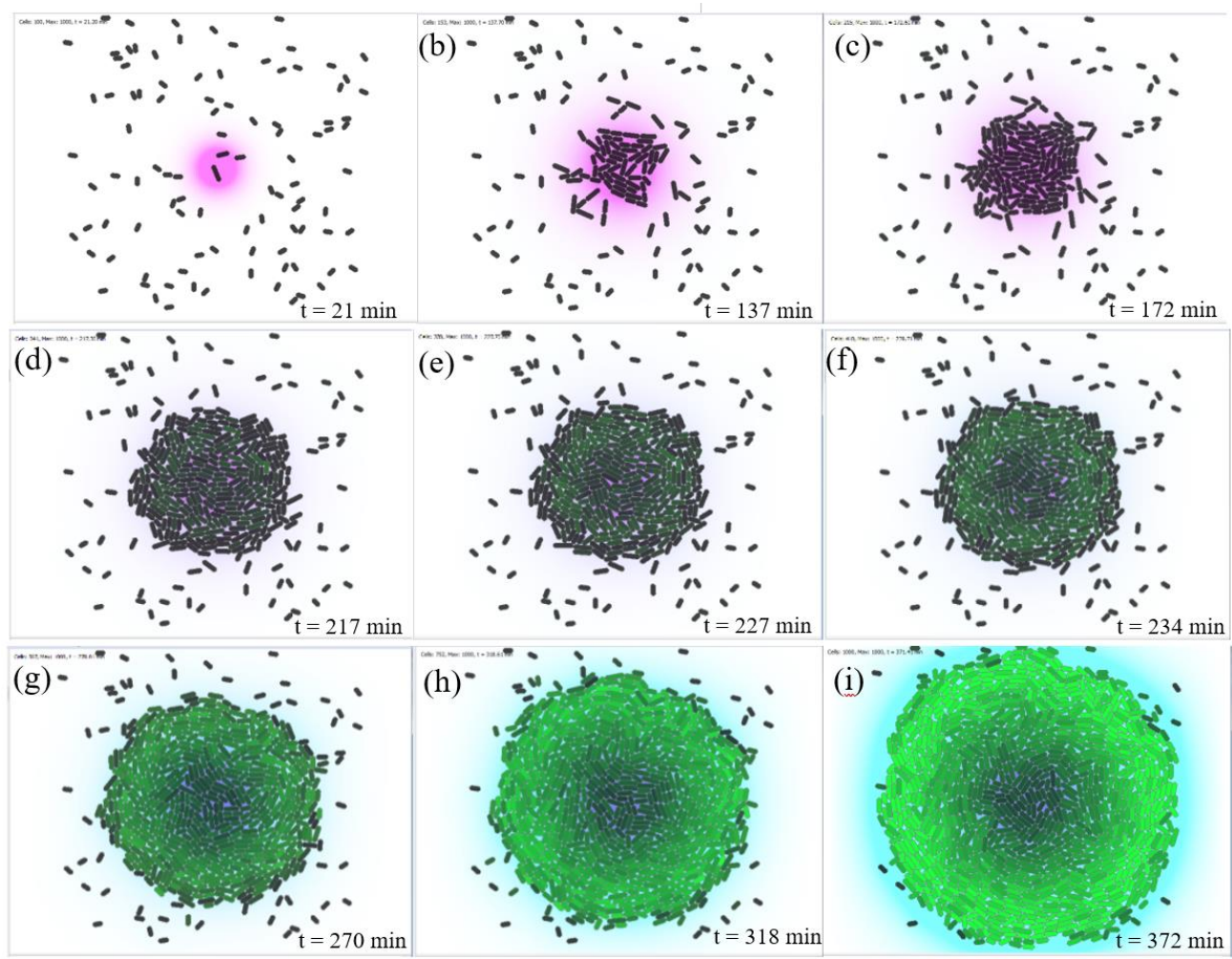

Figure 5: Evolution of bioluminescence, case 1: a single nutrient source placed in the center of the environment. Substrate is shown in purple, $A H L$ in blue, black cells are non-fluorescent. Fluorescence is expressed as a gradient ranging from the dark to light green. (a), (b) and (c) represent the beginning of the simulation: cells grow and divide. In (d), (e) and (f), the quorum is being reached, fluorescent cells begin to appear. Finally, (g), (h) and (i) show homogenous behavior of bioluminescent cells.

not produce light at high intensity. So, they are able to send light at a rate $\mathrm{T}_{\mathrm{L}}=0.20$ to sensitive cheaters.

- Nodes with $g f p / v o l u m e=0$ are nonbioluminescent bacteria called cheaters. They may be non-QS cells or QS cells that do not produce light. They are receivers called cheaters because they do not collaborate toward the common goals (producing light and establishing links) but gain benefit from the other cells that can do so.

This classification of nodes based on the intracellular gfp value at a node allows each node to self-select its type without using a centralized process to attribute to each node a specified type.

\section{Experiments and results}

Using an open source simulator [41], we set up a twodimensional environment of size $(80 \mu \mathrm{m}, 80 \mu \mathrm{m})$. At the beginning of the simulation, 100 generic bacterial agents are randomly dispersed in the environment (Figure 5a). A bacterium cell is assumed to be $1 \mu \mathrm{m}$ in diameter and, initially $2 \mu \mathrm{m}$ long. Thus, its initial volume is $V=1.57 \mathrm{fl}$ the cell divides, it gives rise to two cells. One of the cells is chosen arbitrarily to be the mother and the other becomes its daughter. Then, the program running on the mother is copied to the daughter cell.

\subsection{Quorum sensing dynamics}

The quorum is met at $\mathrm{t} \simeq 180 \mathrm{~min}$, when the population size is 250 cells (Figure $5 \mathrm{~d}$ ). From $t=270 \mathrm{~min}$ towards the end of simulation (Figure $5 \mathrm{~g}$ - i), we can clearly see the AHL in blue around the colony that does not appear obviously in the early stages, because the diffusion of signaling molecules is spatially limited and significantly slower than the kinetic dynamics of bacteria. Figure $6 a$ shows the evolution of the average amount of autoinducer inside cells over time (median and interquartile range of 20 independent runs of the simulation). From the beginning of the simulation to $t \simeq 180 \mathrm{~min}$, the accumulation of AHL inside the cells was stable, but after this crucial moment at which the quorum is met, the intracellular amount of AHL begins to accelerate up to 0.6. In our model, unlike the seminal work of [20], the environment is not initialized with AHL. AHL is rather 
(a)



(c)

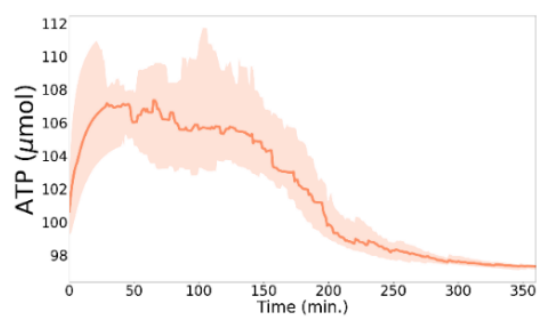

(b)

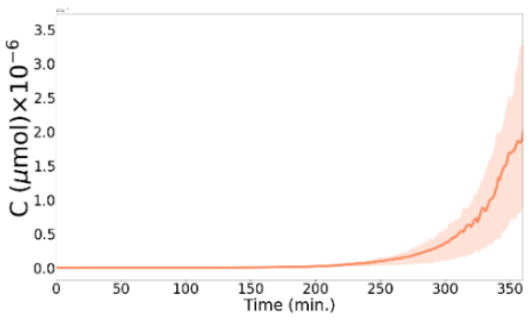

(d)

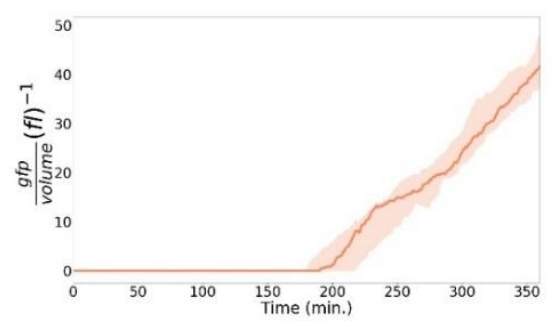

Figure 6: Intracellular molecules dynamics: median and interquartile range of 20 independent simulations. (a) Evolution of the amount of the autoinducer, A. (b) Evolution of the amount of the dimerized complex, C. (c) Evolution of the amount of ATP. (d) Evolution of bioluminescence, gfp/volume.

only produced and diffused by cells. However, in our simulations the average accumulation of $A H L$ by cells exceeds the rate achieved in [20] (0.6 against 0.1$)$. This means that exploiting metabolism, cells grow, reproduce, survive and produce more AHL molecules.

\subsection{Bioluminescence regulation}

Bioluminescence is shown as a gradient ranging from dark to light green. At high cell densities, the number of cells exceeding 250 contributes to the increase of $A H L$ emitted by all the cells in the environment. The cells accelerate the production rates of AHL, using the positive feedback loop in Eq. 1. Consequently, the intracellular amount of the dimerized complex $C$ increases as well, which can be seen in Figure 6b, where the quantity of the dimerized complex begins to increase from $t \simeq 180 \mathrm{~min}$. At this moment, the values of the light production rate given by Eq.12 are positives, and thus cells can accumulate $g f p s$ and express bioluminescence. Indeed, bioluminescence is observed from time $t=217 \mathrm{~min}$ in Figure 5d. In Figure 6d, once the quorum is met at $t \simeq$ $180 \mathrm{~min}$, the cells begin to produce $g f p$. At this precise moment of the simulation, the ATP rate starts to decelerate (Figure 6c). This is due to the energy cost of bioluminescence. In Figure $6 \mathrm{c}$, from $t=300 \mathrm{~min}$ to the end of the simulation, the ATP level is stabilized, which leads to the sustainable behavior of bacteria.

\subsection{Bioluminescence behavior}

It is interesting to observe bioluminescence behavior at the individual and the population level. At the individual level, the number of bioluminescent cells represents $73 \%$ of the population. This corresponds to the empirical rates found in real populations of bacteria. In fact, analysis of the QS-regulated bioluminescence of a wild type strain revealed that only $69 \%$ of the cells of the population produced bioluminescence, $25 \%$ remained dark and $6 \%$ were dead [42].
At the beginning of the simulation, in Figure 5a-f, the arrangement or spatial organization of bioluminescent cells is not homogeneous, and we cannot observe an organized behavior at the population level. However, from $t=318 \mathrm{~min}$, we can clearly see the degradation of the fluorescence from the center of the colony to its border (Figure 5h). The bioluminescent cells organize themselves around the edge of the colony to make other cells emit light. To investigate this hypothesis, in the second case of the simulation, two nutrient sources were placed in the opposite corners of the environment. This allows the development of two colonies as far as possible from each other. We can observe through this simulation in Figure $7 \mathrm{e}-\mathrm{f}$, that the hypothesis is verified. Indeed, in each colony, the bioluminescence occurs toward each other. Also, we can observe that other cells that are not part of both colonies have become fluorescent (green cells surrounded by circles in Figure 7e and 7f).

At the beginning of the simulation, in Figure $5 \mathrm{a}-\mathrm{f}$, the arrangement or spatial organization of bioluminescent cells is not homogeneous, and we cannot observe an organized behavior at the population level. However, from $t=318 \mathrm{~min}$, we can clearly see the degradation of the fluorescence from the center of the colony to its border (Figure 5h). The bioluminescent cells organize themselves around the edge of the colony to make other cells emit light. To investigate this hypothesis, in the second case of the simulation, two nutrient sources were placed in the opposite corners of the environment. This allows the development of two colonies as far as possible from each other. We can observe through this simulation in Figure $7 \mathrm{e}-\mathrm{f}$, that the hypothesis is verified. Indeed, in each colony, the bioluminescence occurs toward each other. Also, we can observe that other cells that are not part of both colonies have become fluorescent (green cells surrounded by circles in Figure 7e and 7f). 


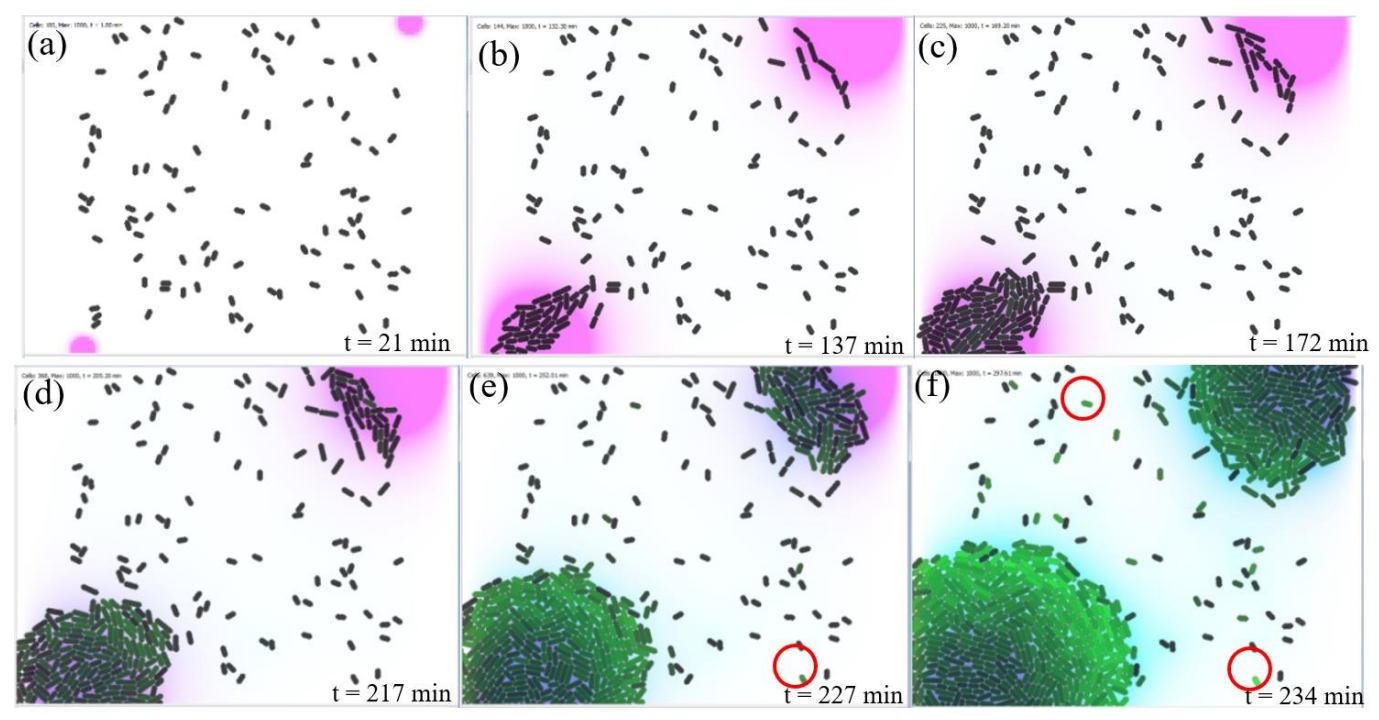

Figure 7: Evolution of bioluminescence-case 2: two nutrient sources, the first placed in the top right of the environment, the second placed in bottom left. (a) and (b): Beginning of the simulation. (c) and (d): Bioluminescent cells begin to appear at the left bottom of the environment. (e) and (f): A self-organized bioluminescence behavior of each colony toward each other.

\subsection{Regeneration and resistance abilities}

The regeneration abilities of our model are tested in two cases: 1) in the light of a random cell death, and 2) a selected area for cell death.

\subsubsection{Random cell death}

In this experiment, we want to verify how the model behaves with a random distributed cell death. In Figure 8, random cell death of 216 cells is inflicted on the bacteria colony at $t=255 \mathrm{~min}$. The $216 \mathrm{dead}$ cells are regenerated at $t=311 \mathrm{~min}$, for a regeneration total time of $56 \mathrm{~min}$. Several simulations of random cells death were carried out over several time stages by varying the number of killed cells.
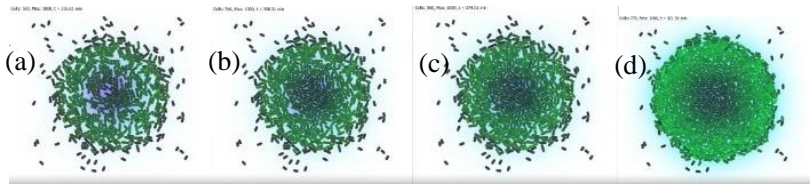

Figure 8: Regeneration after a programed random cell death. (a) a random cell death of 216 cells. (b) and (c) regrowth and regeneration of death cells. (d) Reformation of the colony structure.

Experiments show that the model provides intrinsic abilities of regeneration and maintaining of the structure without reprogramming or adding any further specific technique to the basic model. The average regeneration rate is 4 cells/min for an average regeneration time of $0.25 \mathrm{~min} / \mathrm{cell}$.

\subsubsection{A selected area for cell death}

In this experiment, we want to verify how the model behaves with non-equally distributed cell death, i.e. where a cluster of adjacent cells dies simultaneously due, for example, to the action of an antibiotic or damage on a specific area of the colony structure. To do so, a selected area for cell death is made. In the experiment reported by Figure 9; 26\% of cells of the colony, 124 cells, are killed. Dead cells are regenerated in $34 \mathrm{~min}$. The experiment shows that the model is capable of regrowth, reformation and maintaining of the structure. Several cuts of selected regions for cell death have been tested. The average regeneration rate is 5 cells/min for an average regeneration time of $0.2 \mathrm{~min} /$ cell.



Figure 9: Regeneration after selected area for cell death. (a) A vertical cut (124 killed cells) is made in the middle of the colony. (b)-(c): Regrowth and regeneration of death cells. (d) Reformation of the colony structure.

In [28], after killing $23 \%$ of the artificial multicellular creature, the average regeneration rate is $25 \mathrm{cells} / \mathrm{min}$ for an average regeneration time of $0.04 \mathrm{~min} / \mathrm{cell}$. This represents $1 / 5$ of the regeneration time of our model i.e. $0.2 \mathrm{~min} / \mathrm{cell}$. Despite this observation, we consider a regeneration time between 0.2 to $0.25 \mathrm{~min} / \mathrm{cell}$ as advantageous because our model includes physical force and growth kinetic simulations.

\subsection{Network evolution}

The artificial network evolves as the bacteria colony grows. It evolves via (1) self-selection of the cell type (local test of the $g f p$ value), and (2) sending of links (based on two simple rules, Section 4.1) without calculation of the cell positions. In this section, we want to observe the evolution of the properties of this network, mainly: the number of networked cells and links of each cell type. Also, we need to test whether a fairly simple network definition allows the entire population of cells to be 




(a)

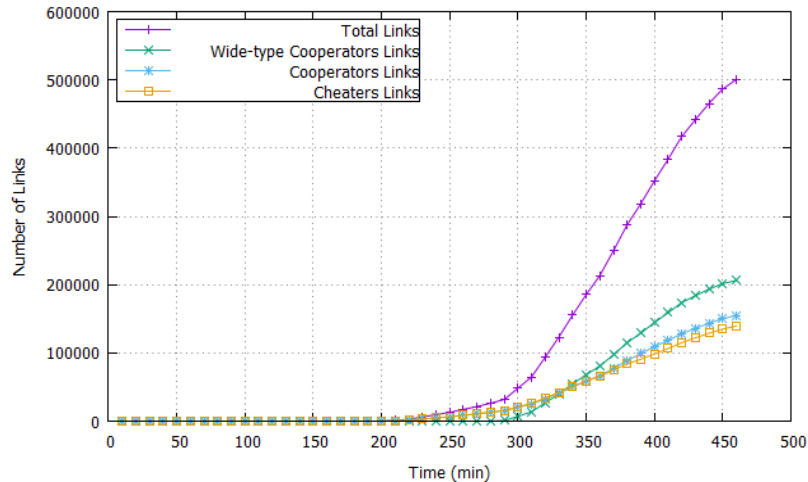

(b)

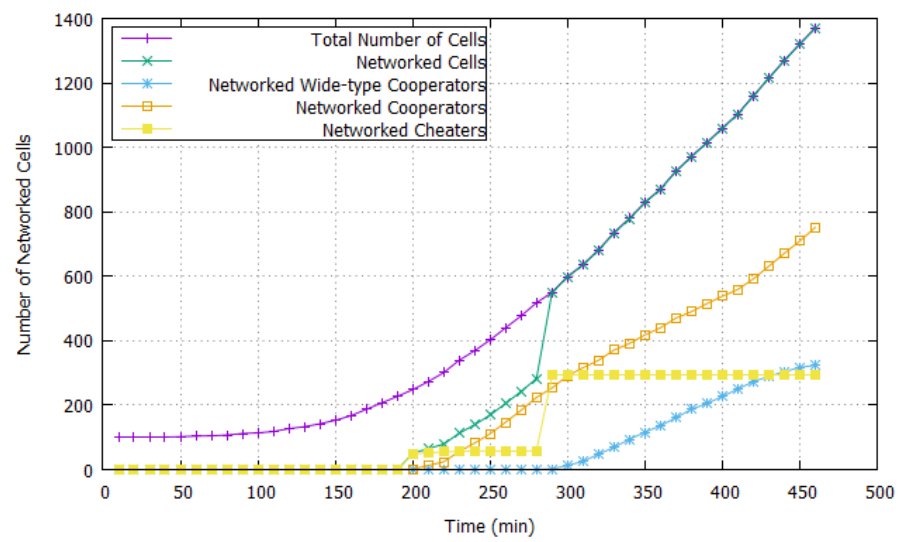

(c)

Figure 10: Measures of the artificial communication network. (a) Evolution of the number of cells. (b) Evolution of the number of links. (c) Evolution of the number of networked cells.

networked (total number of cells $=$ total number of networked cells). To do so, three measures have been considered: number of cells, number of links, and number of networked cells of each cell type.

A cell is considered to be sensitive to light if its intracellular concentration of $l u x R$ is above the threshold $T_{-} R=0.0155$. The first measure we calculate is the numbers of different cell types. This is represented in Figure 10a. In Figure 10a, from $t=0 \mathrm{~min}$ to $t=$ $50 \mathrm{~min}$, the number of cheaters is stable and equal to 100 cells. As the colony grows, the number of cheaters begins to increase gradually, where it reaches its maximum i.e. 294 cells at $t=260 \mathrm{~min}$. After this time, the number stagnates until the end of the simulation. At the instant $t=210 \mathrm{~min}$, the quorum is being met, the cells begin to emit light and consequently cooperators begin to emerge in the population. At $t=300 \mathrm{~min}$ the number of cooperators exceeds the number of cheaters, while WTcooperators start to appear. The number of cooperators and WT-cooperators continues to increase as the number of cells increases, while the number of cheaters remains stable. At the end of the simulation, we can notice that cooperator cells are dominant in the population with 752 cells which represents $55 \%$ of the population, compared to 294 cheaters and 324 WT-cooperators which account for $21 \%$ and $24 \%$, respectively.

Figure 10c represents the third measure we calculate, the number of networked cells. At $t=290 \mathrm{~min}$, networked WT-cooperator cells begin to emerge and the number of networked cells fit the total number of total cells (the number of non-networked cells is equal to $0)$. Consequently, the network model permits all cells of the population to be networked without calculating the cell position. Indeed, the assumption we have made in the beginning of this section is verified. Although our communication network is defined on the basis of two simple rules, an emission rule and a reception rule, the network allows the totality of the cells of the population $(100 \%)$ to be networked, whatever their position (near or far from the colony) or their type (QS or non-QS cell, bioluminescent or non-bioluminescent cell).

We note that the results converged as a spontaneous evolution of the network dynamics without the use of any evolutionary algorithm. The artificial network has also the property of self-selection of the node's type without a centralized control. Such artificial communication networks can be used for the emergence of self-organizing wireless network topologies that address issues such as location data and overhead messages.

\subsection{Cooperation measurements}

As in macroscale communities, within microbiomes, different types of bacteria tend to cooperate towards common goals. Since nodes and links are basic components for any network topology, we consider two common goals. The first common goal is the evolution of 
the total number of links. The second common goal is the evolution of the number of networked cells.

Our artificial communication network is based on a cooperative behavior of QS which is light. This cooperative behavior determines the network's node types (via the $g f p$ value) and how the links are established (a descending gradient of light). So, we need to measure how much cooperator cells contribute towards the common goals for the network: links and nodes. Also, we want to determine which kind of cooperators has the highest degree of cooperation for the common goals. To do so, cooperation is calculated as the benefit/cost ratio. Table 1 shows the results considering two measures of cooperation. The first measure describes the contribution of cooperators (respectively WT-cooperators) for the common goal number 1: total number of links. The second measure describes the contribution of cooperators (respectively WT-cooperator) for the goal number 2: total number of networked cells.

\begin{tabular}{|c|c|c|}
\hline Node type & $\begin{array}{c}\text { Common goal 1: } \\
\text { total links }\end{array}$ & $\begin{array}{c}\text { Common goal } \\
2: \text { networked } \\
\text { cells }\end{array}$ \\
\hline Cooperators & $500000 / 752=664.89$ & $1370 / 752=1.82$ \\
\hline $\begin{array}{c}\text { WT- } \\
\text { cooperators }\end{array}$ & $500000 / 324=1543.20$ & $1370 / 324=4.22$ \\
\hline
\end{tabular}

Table 2: Cooperation measurements of the network.

The total number of links is 50000, the number of networked cells is 1370 , the number of cooperators is 752 and the number WT-cooperators is 324 . We note that upregulated cells, here WT-cooperators, have the higher degree of cooperation in the evolution of the properties of the subsequent communication network.

\section{Discussion}

To observe interesting behaviors of bacteria, it is necessary to simulate the interactions of a large number of bacterial cells in in silico models. Our model enables the colonies to have up to 1400 bacterial cells, while in the seminal work presented in [20] the population size does not exceed 256 cells, and 430 cells in [9]. We proposed a simplified computational model for bioluminescence. Nevertheless, bioluminescence emerges as a spontaneous property of the regulation system, without any centralized control on the QS genetic circuit. We note that a cell counter to measure cell density is not used to control QS, and neither global or local control is applied to cellular actions. In [9], the authors propose an algorithm of QS that uses a cell counter, to decide the behavior of cells. Conversely, in our model, cell actions are executed autonomously by the bacterial agent, all the time and in parallel-similarly to the reactions of real cells.

In most artificial life models, metabolism is rarely taken into account, or it is greatly abstracted into a simplistic model where the amount of energy decreases at each time step, as in [9], [43], [44]. In such models, there is no real transformation of matter from nutrients to biomass, or ATP. However, in our model, there is an actual simulation of such production and consumption of energy. Positive terms in the metabolism equation (Eq. 13) describe energy production (transformation of matter, i.e. metabolization of substrate into ATP and biomass), while negative terms describe energy consumption. The control of metabolism is one of the important features of QS regulation [45]. It is notable that our research is one of the few studies which link metabolism to QS. QS-regulated microbial metabolism includes bioenergy production [46], resource utilization and energy optimization, which are essential to population survival [47]. The results presented in this paper showed that metabolism has been regulated i.e. stabilized after the quorum is being met. This feature is very important for regulating the battery lifetime i.e. survival of the proposed artificial wireless networks.

\section{Conclusion}

We proposed computational models of metabolism and bioluminescence, allowing artificial bacterial agents to produce their own energy and communicate using light signals. We have also presented a self-sustainable network model in which the rules governing the formation of the network are linked to the dynamics of its components without any centralized control. Results show regeneration abilities and the emergence of homogenous behaviors over the population, e.g. the cooperation toward common goals in the evolution of the artificial communication network. This cooperation allows the totality of the cells of the population to be networked. The resulting artificial network could be potentially used for the emergence of wireless network topologies without the use of overhead messages and with self-maintaining and resistance features.

Finally, pathogen microscopic bacteria in nature develop efficient and secure communication networks using the bacterial communication consensus (i.e. Quorum Sensing), which is highly robust to external attacks, displaying drug or antibiotic resistance. Quenching microbial quorum sensing, known as "Quorum quenching", is a strategy to inhibit the QS dynamics and thus the "bugs" that can be caused by pathogen bacteria. This concept could be used to prevent the security issue and develop self-organized defense mechanisms based on our QS-based artificial wireless networks. Additionally, our network could also be evolved with an evolutionary algorithm to solve specific common networks problems such as energy optimization or improvement of the quality of the network links. We hope that our work on QS inspired artificial wireless networks can foster ideas for future investigations.

\section{References}

[1] T. E. Gorochowski, "Agent-based modelling in synthetic biology," Essays Biochem., vol. 60, no. 4, pp. 325-336, 2016. https://doi.org/10.1042/EBC20160037

[2] B. L. Bassler, "How bacterial talk to each other: regulation of gene expression by quorum sensing," Curr. Opin. Microbiol., vol. 2, no. 6, pp. 582-587, 1999. 
https://doi.org/10.1016/S1369-5274(99)00025-9

[3] K. O. Stanley and R. Miikkulainen, "A Taxonomy for Artificial Embryogeny,” Artif. Life, vol. 9, no. 2, pp. 93-130, 2003. https://doi.org/10.1162/106454603322221487

[4] R. Doursat, H. Sayama, and O. Michel, "A review of morphogenetic engineering," Nat. Comput., vol. 12, no. 4, pp. 517-535, 2013. https://doi.org/10.1007/s11047-013-9398-1

[5] A. Chavoya and Y. Duthen, "A cell pattern generation model based on an extended artificial regulatory network," Biosystems, vol. 94, no. 1-2, pp. 95-101, 2008. https://doi.org/10.1016/j.biosystems.2008.05.015

[6] S. Nichele, T. E. Glover, and G. Tufte, "Genotype Regulation by Self-modifying Instruction-Based Development on Cellular Automata," Springer, Cham, 2016, pp. 14-25.

https://doi.org/10.1007/978-3-319-45823-6_2

[7] S. Majumdar and S. Mondal, "Conversation game: talking bacteria," J. Cell Commun. Signal., vol. 10, no. 4, pp. 331-335, 2016. https://doi.org/10.1007/s12079-016-0333-y

[8] F. Dressler and O. B. Akan, "A survey on bioinspired networking," Comput. Networks, vol. 54, no. 6, pp. 881-900, 2010. https://doi.org/10.1016/j.comnet.2009.10.024

[9] N. Ouannes, N. Djedi, Y. Duthen, and H. Luga, "Emergent group behaviors from bacteria quorum sensing simulation," in 21st AROB, 2016, pp. 6267.

[10] F. J. Romero-Campero and M. J. Pérez-Jiménez, “A Model of the Quorum Sensing System in Vibrio fischeri Using P Systems," Artif. Life, vol. 14, no. 1, pp. 95-109, 2008. https://doi.org/10.1162/artl.2008.14.1.95

[11] B. E. Beckmann and P. K. Mckinley, "Evolving Quorum Sensing in Digital Organisms," in GECCO'09 Proceedings of the 11th Annual conference on Genetic and evolutionary computation, 2009, pp. 97-104. https://doi.org/10.1145/1569901.1569916

[12] P. Bechon and J.-J. Slotine, "Synchronization and quorum sensing in a swarm of humanoid robots," arXiv Prepr. arXiv1205.2952, 2012.

[13] W. Ji et al., “A Formalized Design Process for Bacterial Consortia That Perform Logic Computing," PLoS One, vol. 8, no. 2, p. e57482, 2013. https://doi.org/10.1371/journal.pone.0057482

[14] Feng Tan and J.-J. Slotine, "A quorum sensing inspired algorithm for dynamic clustering," in 52nd IEEE Conference on Decision and Control, 2013, pp. 5364-5370 https://doi.org/10.1109/CDC.2013.6760733

[15] H. Shum and A. C. Balazs, "Synthetic quorum sensing in model microcapsule colonies," Proc. Natl. Acad. Sci., vol. 114, no. 32, pp. 8475-8480, 2017. https://doi.org/10.1073/pnas.1702288114

[16] B. Niu, H. Wang, Q. Duan, and L. Li, "Biomimicry of quorum sensing using bacterial lifecycle model,"
BMC Bioinformatics, vol. 14, no. Suppl 8, p. S8, 2013.

https://doi.org/10.1186/1471-2105-14-S8-S8

[17] D. A. Sofge and W. F. Lawless, "Quorum Sensing for Collective Action and Decision-Making in Mobile Autonomous Teams," in ICAART (1), 2011, pp. 195-204.

[18] A. C. Burgos and D. Polani, "Cooperation and antagonism in information exchange in a growth scenario with two species," J. Theor. Biol., vol. 399 pp. 117-133, 2016. https://doi.org/10.1016/j.jtbi.2016.04.006

[19] J. W. Williams, X. Cui, A. Levchenko, and A. M. Stevens, "Robust and sensitive control of a quorumsensing circuit by two interlocked feedback loops," Mol. Syst. Biol., vol. 4, no. 1, p. 234, 2008. https://doi.org/10.1038/msb.2008.70

[20] P. Melke, P. Sahlin, A. Levchenko, and H. Jönsson, "A cell-based model for quorum sensing in heterogeneous bacterial colonies," PLoS Comput. Biol., vol. 6, no. 6, p. e1000819, 2010. https://doi.org/10.1371/journal.pcbi.1000819

[21] G. Wei, C. Walsh, I. Cazan, and R. Marculescu, "Molecular tweeting: Unveiling the social network behind heterogeneous bacteria populations," in BCB 2015 - 6th ACM Conference on Bioinformatics, Computational Biology, and Health Informatics, 2015, pp. 366-375. https://doi.org/10.1145/2808719.2808757

[22] A. A. Aziz, Y. A. Sekercioglu, P. Fitzpatrick, and M. Ivanovich, "A Survey on Distributed Topology Control Techniques for Extending the Lifetime of Battery Powered Wireless Sensor Networks," IEEE Commun. Surv. Tutorials, vol. 15, no. 1, pp. 121144, 2013. https://doi.org/10.1109/SURV.2012.031612.00124

[23] J. F. Miller, "Evolving Developmental Programs for Adaptation, Morphogenesis, and Self-Repair," in European Conference on Artificial Life., 2004, pp. 256-265. https://doi.org/10.1007/978-3-540-39432-7_28

[24] J. F. Miller, "Evolving a Self-Repairing, SelfRegulating, French Flag Organism," in Genetic and Evolutionary Computation Conference, 2004, pp. 129-139. https://doi.org/10.1007/978-3-540-24854-5_12

[25] H. Liu, J. F. Miller, and A. M. Tyrrell, “An intrinsic robust transient fault-tolerant developmental model for digital systems," in Workshop on Regeneration and Learning in Developmental Systems, Genetic and Evolutionary Computation Conference., 2004.

[26] K. Fleischer, "Investigations with a Multicellular Developmental Model," in C. G. Langton \& T. Shimohara (Eds.), Artificial Life V, 1996, pp. 229236.

[27] S. Cussat-Blanc, H. Luga, and Y. Duthen, "Cell2Organ: Self-repairing artificial creatures thanks to a healthy metabolism," in 2009 IEEE Congress on Evolutionary Computation, CEC 2009, 2009, pp. 2708-2715. https://doi.org/10.1109/CEC.2009.4983282 
[28] N. Djezzar, N. Djedi, S. Cussat-Blanc, H. Luga, and Y. Duthen, "L-systems and artificial chemistry to develop digital organisms," in 2011 IEEE Symposium on Artificial Life (ALIFE), 2011, pp. 225-232. https://doi.org/10.1109/ALIFE.2011.5954665

[29] G. Ferreira, M. Smiley, M. Scheutz, and M. Levin, "Dynamic Structure Discovery and Repair for 3D Cell Assemblages," in Proceedings of the Artificial Life Conference 2016, 2016, pp. 352-359. https://doi.org/10.7551/978-0-262-33936-0-ch059

[30] L. Cobo, A. Quintero, and S. Pierre, "Ant-based routing for wireless multimedia sensor networks using multiple QoS metrics," Comput. Networks, vol. 54, no. 17, pp. 2991-3010, 2010. https://doi.org/10.1016/j.comnet.2010.05.014

[31] A. Giagkos and M. S. Wilson, "Swarm intelligence to wireless ad hoc networks: adaptive honeybee foraging during communication sessions," Adapt. Behav., vol. 21, no. 6, pp. 501-515, 2013. https://doi.org/10.1177/1059712313500797

[32] I. Bojic, V. Podobnik, I. Ljubi, G. Jezic, and M. Kusek, "A self-optimizing mobile network: Autotuning the network with firefly-synchronized agents," Inf. Sci. (Ny)., vol. 182, no. 1, pp. 77-92, 2012.

https://doi.org/10.1016/j.ins.2010.11.017

[33] Z. G. Al-Mekhlafi, Z. M. Hanapi, M. Othman, and Z. A. Zukarnain, "A firefly-inspired scheme for energy-efficient transmission scheduling using a self-organizing method in a wireless sensor networks," J. Comput. Sci., vol. 12, no. 10, pp. 82 494, 2016. https://doi.org/10.3844/jessp.2016.482.494

[34] N. El Houda Bahloul, S. Boudjit, M. Abdennebi, and D. E. Boubiche, "A Flocking-Based on Demand Routing Protocol for Unmanned Aerial Vehicles," J. Comput. Sci. Technol., vol. 33, no. 2, pp. 263-276, 2018.

https://doi.org/10.1007/s11390-018-1818-3

[35] J. Monod, "The Growth of Bacterial Cultures," Annu. Rev. Microbiol., vol. 3, no. 1, pp. 371-394, 1949.

https://doi.org/10.1146/annurev.mi.03.100149.0021 03

[36] A. H. Stouthamer and C. Bettenhaussen, "Utilization of energy for growth and maintenance in continuous and batch cultures of microorganisms. A reevaluation of the method for the determination of ATP production by measuring molar growth yields," BBA Reviews On Bioenergetics, vol. 301, no. 1. pp. 53-70, 1973. https://doi.org/10.1016/0304-4173(73)90012-8

[37] S. A. L. M. Kooijman, Dynamic energy budget theory for metabolic organisation. Cambridge University Press, 2010. https://doi.org/10.1017/CBO9780511805400

[38] A. I. Psarras and I. G. Karafyllidis, "Simulation of the Dynamics of Bacterial Quorum Sensing," IEEE Trans. Nanobioscience, vol. 14, no. 4, pp. 440-446, Jun. 2015.
https://doi.org/10.1109/TNB.2014.2385109

[39] D. J. Sexton and M. Schuster, "Nutrient limitation determines the fitness of cheaters in bacterial siderophore cooperation," Nat. Commun., vol. 8, no. 1, p. 230, 2017. https://doi.org/10.1038/s41467-017-00222-2

[40] C. Moreno-Fenoll, M. Cavaliere, E. MartínezGarcía, and J. F. Poyatos, "Eco-evolutionary feedbacks can rescue cooperation in microbial populations," Sci. Rep., vol. 7, p. 42561, 2017. https://doi.org/10.1038/srep42561

[41] S. S. Jang, K. T. Oishi, R. G. Egbert, and E. Klavins, "Specification and Simulation of Synthetic Multicelled Behaviors," ACS Synth. Biol., vol. 1, no. 8, pp. 365-374, 2012. https://doi.org/10.1021/sb300034m

[42] C. Anetzberger, T. Pirch, and K. Jung, "Heterogeneity in quorum sensing-regulated bioluminescence of Vibrio harveyi," Mol. Microbiol., vol. 73, no. 2, pp. 267-277, 2009. https://doi.org/10.1111/j.1365-2958.2009.06768.x

[43] N. Ouannes, N. Djedi, H. Luga, and Y. Duthen, "Modeling a bacterial ecosystem through chemotaxis simulation of a single cell," Artif. Life Robot., vol. 19, no. 4, pp. 382-387, 2014. https://doi.org/10.1007/s10015-014-0187-4

[44] S. Forrest and T. Jones, "Modeling Complex Adaptive Systems with Echo," in Complex Systems: Mechanisms of Adaptation, 1993, pp. 3-21.

[45] S. Y. Queck et al., "RNAIII-independent target gene control by the agr quorum- sensing system: insight into the evolution of virulence regulation in Staphylococcus aureus," Mol. Cell, vol. 32, no. 1, pp. 150-158, 2008.

https://doi.org/10.1016/j.molcel.2008.08.005

[46] Y.-C. Yong and J.-J. Zhong, "Impacts of Quorum Sensing on Microbial Metabolism and Human Health," in Advances in biochemical engineering/biotechnology, vol. 131, 2012, pp. 2561. https://doi.org/10.1007/10_2012_138

[47] E. Goo, J. H. An, Y. Kang, and I. Hwang, "Control of bacterial metabolism by quorum sensing," Trends in Microbiology, vol. 23, no. 9. 2015. https://doi.org/10.1016/j.tim.2015.05.007 
